\title{
Performance Analysis of Joint Diversity Combining, Adaptive Modulation, and Power Control Schemes
}

\author{
Khalid A. Qaraqe, Senior Member, IEEE, Zied Bouida, Student Member, IEEE, \\ and Mohamed-Slim Alouini, Fellow, IEEE
}

\begin{abstract}
Adaptive modulation and diversity combining represent very important adaptive solutions for future generations of wireless communication systems. Indeed, in order to improve the performance and the efficiency of these systems, these two techniques have been recently used jointly in new schemes named joint adaptive modulation and diversity combining (JAMDC) schemes. Considering the problem of finding low hardware complexity, bandwidth-efficient, and processing-power efficient transmission schemes for a downlink scenario and capitalizing on some of these recently proposed JAMDC schemes, we propose and analyze in this paper three joint adaptive modulation, diversity combining, and power control (JAMDCPC) schemes where a constant-power variable-rate adaptive modulation technique is used with an adaptive diversity combining scheme and a common power control process. More specifically, the modulation constellation size, the number of combined diversity paths, and the needed power level are jointly determined to achieve the highest spectral efficiency with the lowest possible processing power consumption quantified in terms of the average number of combined paths, given the fading channel conditions and the required bit error rate (BER) performance. In this paper, the performance of these three JAMDCPC schemes is analyzed in terms of their spectral efficiency, processing power consumption, and error-rate performance. Selected numerical examples show that these schemes considerably increase the spectral efficiency of the existing JAMDC schemes with a slight increase in the average number of combined paths for the low signal-to-noise ratio range while maintaining compliance with the BER performance and a low radiated power which yields to a substantial decrease in interference to co-existing users and systems.
\end{abstract}

Index Terms-Diversity techniques, minimum selection generalized selection combining, adaptive modulation, power control, Rayleigh fading channels, performance analysis.

\section{INTRODUCTION}

A DAPTIVE modulation [1], [2], adaptive diversity combining techniques [3], [4], and power control [5], [6]

Paper approved by N. C. Beaulieu, the Editor for Wireless Communication Theory of the IEEE Communications Society. Manuscript received October 5, 2009; revised February 11, 2010 and June 7, 2010.

This is an expanded version of work which was presented at the IEEE Vehicular Technology Conference (VTC Spring'2009), Barcelona, Spain, April 2009. This work is supported by Qatar National Research Fund (QNRF) grant through National Priority Research Program (NPRP) No. NPRP 29-67-4. QNRF is an initiative of Qatar Foundation.

K. A. Qaraqe is with the Department of Electrical and Computer Engineering, Texas A\&M University at Qatar, Education City, Doha, Qatar (e-mail: khalid.qaraqe@qatar.tamu.edu).

Z. Bouida is a Ph.D. student with the Department of Electrical and Computer Engineering, Texas A\&M University, College Station, Texas, USA (e-mail: bouidazied@tamu.edu).

M.-S. Alouini is with the Electrical Engineering Program, KAUST, Thuwal, Saudi Arabia (e-mail: slim.alouini@kaust.edu.sa).

Digital Object Identifier 10.1109/TCOMM.2010.111710.090600 are getting more and more importance in modern wireless communication systems. Many reasons are behind the use of these key adaptive solutions. Indeed, future wireless communication systems which will provide multimedia services to the power limited mobile terminals are characterized by limited bandwidth and power resources. These systems should be able to support high spectral efficiency with good link reliability. This need for higher bandwidth efficiency motivates further optimization of the use of wireless resources. Due to user mobility and highly time-variant propagation environments, resource management in wireless communications becomes a difficult task. In order to facilitate the management of these resources, adaptive techniques seem to be one of the best solutions.

Based on multiple thresholds, adaptive modulation can achieve high spectral efficiency over wireless channels. The key idea of adaptive modulation is to adapt the modulation parameters, such as constellation size, to fading channel conditions while respecting the bit error rate (BER) requirements. Diversity combining, on the other hand, improves the reliability of wireless fading channels by adapting the combiner structure to fading channel conditions. Adaptive power control schemes, unlike schemes using a constant-power variablerate setup, adapt the transmitted power to fading channels conditions while fulfilling the BER constraint. These schemes reduce the radiated power, and thus the potential interference to other users which implies a significant network capacity improvements.

Generalized selection combining (GSC) is one of diversity combining schemes that received a great deal of attention over the last decade [7]-[9]. The receiver with GSC combines a fixed number of best diversity paths as per the rules of the optimal maximum ratio combining (MRC) scheme. The hardware complexity of GSC, quantified in terms of the number of branches implemented in the combiner, is then minimized compared to the hardware complexity of the MRC combiner. Minimum selection GSC (MS-GSC) was proposed in [10] as a power-saving implementation of GSC. With MSGSC the receiver ranks the signal-to-noise ratio (SNR) of all available paths and then combines the minimum number of branches in order to make the combined SNR exceed a certain predetermined threshold. On average, MS-GSC combines less branches and hence uses less processing power which can be quantified in terms of the average number of combined paths [10], [11], making it ideal for a downlink scenario where the mobile unit is power and size limited. 
These adaptive solutions have been originally studied separately. Recently, joint adaptive solutions have been proposed and studied. For instance, while joint adaptive modulation and diversity combining schemes were introduced in [12], joint adaptive combining and power control were studied for constant-rate transmission in [13]. In addition, in [14] and for the purpose of interference reduction, Gjendemsjø et. al. extended the schemes discussed in [12], [13] by looking at joint adaptive modulation, diversity combining, and postcombining power control (JAMDC). In these JAMDC schemes the receiver jointly determines the most appropriate modulation mode and diversity combiner structure based on the current channel conditions and the desired BER requirements. Capitalizing on this recent work and on the work done in [12], and in order to have better spectral efficiency, better BER performance, and less radiated power, we offer in this paper a generalization of the existing schemes by proposing three joint adaptive modulation, diversity combining, and power control schemes (JAMDCPC), namely (i) a processing power efficient scheme (JAMDCPC-1), (ii) a bandwidth efficient scheme (JAMDCPC-2), and (iii) a bandwidth efficient with finger deactivation scheme (JAMDCPC-3). While the JAMDC schemes proposed in [14] apply power control in the end of the combining process, our schemes use a joint power control process that allows to increase or decrease the transmitted power depending on the value of the combined SNR. The three proposed schemes have different optimization problems that depend on the primary objective of the joint design of adaptive modulation, diversity combining, and power control. While JAMDCPC-1 tends to use the minimum number of combined paths that allows to reach the lowest possible modulation mode, JAMDCPC-2 aims to use the highest constellation size regardless of how many branches are activated in the receiver's side. As a balance between these two schemes, JAMDCPC-3 aims to reach the highest modulation mode while applying a finger deactivation (FD) process that reduces the processing power consumption expressed in terms of the average number of combined paths. We analyze these newly proposed schemes in terms of average spectral efficiency (ASE), average BER, processing power consumption, and transmitted power gain and compare their performance to that of the processing power efficient scheme (PES-JAMDC) and the bandwidth efficient scheme (BES-JAMDC) schemes proposed in [14] and to that of the bandwidth-efficient and power-greedy scheme proposed in [12]. Selected numerical examples, obtained by Monte Carlo simulations and confirmed by analytical results, show that the proposed JAMDCPC schemes (i) have better ASE with a slight increase in the average number of combined paths, (ii) improve the BER performance, and (iii) maintain a low average radiated power yielding to a substantial decrease in interference to co-existing users and systems.

The remainder of the paper is organized as follows. Section II presents first the system and channel models, then gives the details behind the adaptive transmission system, and the underlying power control mechanism. Section III gives a detailed mode of operation of the proposed schemes and the statistics of the combined SNR before power control. While Section IV analyzes the performance of the JAMDCPC schemes, Section $\mathrm{V}$ offers some selected numerical examples illustrating this

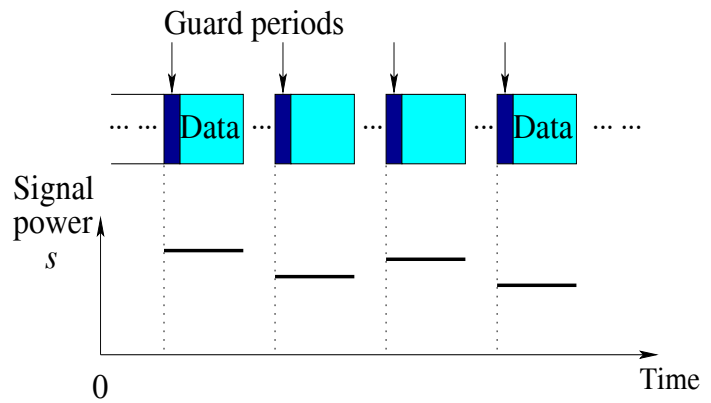

Fig. 1. Block fading channel model.

performance and comparing it to that of existing schemes. Finally, Section VI concludes the paper.

\section{System AND Channel Models}

\section{A. System Model}

We consider a generic diversity system with $L$ available diversity paths. This includes, for example, RAKE receivers which are used in wideband CDMA systems to combine the available resolvable multipaths. For hardware complexity considerations, we assume that up to $L_{c}$ branches can be combined at the receiver side (i.e., the number of fingers of the RAKE receiver is limited to $L_{c}$ ). We also assume that the proposed JAMDCPC schemes have a reliable feedback path between the receiver and the transmitter and are implemented in a discrete-time fashion. More specifically, and as shown in Fig. 1, short guard periods followed by longer data burst are periodically inserted into the transmitted signal. During these guard periods, the receiver performs a series of operations, including (i) path estimation, (ii) combined SNR comparison with respect to the predetermined SNR threshold, and (iii) when needed request to the transmitter high power amplifier (HPA) to increase or decrease its gain by a specific amount. In our proposed schemes the receiver jointly determines the diversity combiner structure, the modulation mode, and the power control level based on the current channel conditions and the desired BER requirements. Once the suitable paths for combining and the suitable modulation mode are selected and once the appropriate transmitted power is reached, the combiner and the HPA are configured accordingly, and this transmitter and receiver settings are used throughout the subsequent data burst.

\section{B. Channel Model}

Under the assumption of multipath channel, we denote by $\gamma_{l}(l=1,2, \ldots, L)$, the received SNR of the $l$ th diversity path under nominal transmitted power from the $\mathrm{BS}^{1}$ and, as illustrated in Fig. 1, we adopt a block flat fading channel model. More specifically, assuming slowly-varying fading conditions, the different diversity paths experience roughly the same fading conditions during the data burst and its preceding guard period. In addition, the fading conditions are assumed

\footnotetext{
${ }^{1}$ The BS nominal transmitted power is assumed to correspond to an initial level of output power that is adjusted or set to minimize the average outer cell interference in a particular deployment.
} 
to (i) be independent across the diversity paths and between different guard period and data burst pairs, and (ii) follow anyone of the popular fading models such as Rayleigh, Rice, or Nakagami- $m$.

For our study, we assume that the multipath envelop of each path follows the Rayleigh fading model. We also assume that the fading signal envelops on all diversity branches are mutually independent and identically distributed (i.i.d.). The probability density function (pdf) $f_{\gamma}(x)$ and the cumulative distribution function (cdf) $F_{\gamma}(x)$ of the faded SNR $\gamma_{i}, i=$ $1, \ldots, L$ for a diversity path for Rayleigh fading model are given by

$$
f_{\gamma_{i}}(x)=\frac{1}{\bar{\gamma}} \exp \left(-\frac{x}{\bar{\gamma}}\right), \quad x \geq 0
$$

and

$$
F_{\gamma_{i}}(x)=1-\exp \left(-\frac{x}{\bar{\gamma}}\right), \quad x \geq 0,
$$

respectively, where $\bar{\gamma}$ is the common average faded SNR which is defined as in [9] by

$$
\bar{\gamma}=\Omega E_{s} / N_{0},
$$

where $\Omega$ is the common average energy gain per path and $E_{s} / N_{0}$ is the symbol energy-to-Gaussian noise spectral density ratio.

\section{Adaptive Transmission System}

We consider the constant-power variable-rate $M$-ary QAM [15] as the adaptive modulation system of choice for our proposed adaptive transceiver. With this adaptive modulator, the SNR range is divided into $N+1$ fading regions and the constellation size $M=2^{n}$, where $n$ is the number of bits per symbol, is assigned to the $n$th region $(n=0,1, \ldots, N)$. The selection of a constellation size is based on the fading channel state. Specifically, we partition the range of the SNR after diversity combining into $N+1$ regions, which are defined by the switching thresholds $\left\{\gamma_{T_{n}}\right\}_{n=1}^{N}$, and the constellation size is selected according to the combined SNR before power control $\Gamma$ and the transmitter gain at saturation $G_{\max }$. If $\Gamma \in\left[\gamma_{T_{n}}, \gamma_{T_{n+1}} / G_{\max }\right)$ then the modulation mode $n$ is used. If on the other hand $\Gamma \in\left[\gamma_{T n+1} / G_{\max }, \gamma_{T n+1}\right)$ then the transmitter will increase its power in order to reach the next constellation size, and the modulation mode $n+1$ is used. ${ }^{2}$

The BER of $2^{n}$-QAM constellations with SNR of $\gamma$ is given in [15] by

$$
P_{b_{n}}(\gamma)=\frac{1}{5} \exp \left[\frac{-3 \gamma}{2\left(2^{n}-1\right)}\right] .
$$

Given a target instantaneous BER equal to $P_{b_{0}}$, the region boundaries (or adaptive modulator switching thresholds) $\gamma_{T_{n}}$ for $n=0,1, \ldots N$ are given in this case by

$$
\gamma_{T n}=-\frac{2}{3} \ln \left(P_{b_{0}}\right)\left(2^{n}-1\right) ; n=0,1, \ldots N .
$$

\footnotetext{
${ }^{2}$ In our simulations we worked with $G_{\operatorname{maxdB}}=1 \mathrm{~dB}$. The main idea is to add power if the receiver needs less than $G_{\operatorname{maxdB}}$ in order to reach the next constellation size. This makes the new schemes have better spectral efficiency with a slight increase in the average radiated power.
}

\section{Power Control}

In our proposed schemes we use the same power control mechanism described here. While in the existing JAMDC schemes the transmitter starts sending a training sequence using the highest available power level, in the JAMDCPC schemes we assume that the transmitter starts sending with the nominal power level which is adjusted or set by the system to minimize the average outer cell interference in a particular deployment. This will allow the HPA not only to decrease the transmitted power but also to increase it in order to reach a higher modulation mode. If the combined SNR before power control falls in $\left[\gamma_{T_{n}}, \gamma_{T_{n+1}}\right)$ then we consider two cases (i) if $\Gamma \in\left[\gamma_{T_{n}}, \gamma_{T_{n+1}} / G_{\max }\right)$ then the HPA will reduce its power while staying in the same modulation mode, (ii) if $\Gamma \in\left[\gamma_{T_{n+1}} / G_{\max }, \gamma_{T_{n+1}}\right)$ then the transmitter will increase its power in order to reach the next constellation size $n+1$.

In an ideal adaptive power control system, we can assume that the transmitter power can be varied continuously to accurately follow channel variations. In the JAMDCPC schemes, in addition to the continuous power adaptation, we also consider power control adaptations accounting for practical implementation constraints including discrete power levels $\left(G_{\delta}\right)$ and a transmitter gain saturation $\left(G_{\max }\right)$.

1) Continuous Power Control: In this first case, we assume that the gain $G$ of the HPA can be adjusted in a continuous fashion. In the beginning of each data burst the transmitter $\mathrm{dB}$ gain $G_{\mathrm{dB}}$ is initially set to $0 \mathrm{~dB}$ with respect to the nominal transmitted power. If $\Gamma \in\left[\gamma_{T_{n}}, \gamma_{T_{n+1}} / G_{\max }\right)$ then the HPA reduces its power by $\Gamma / \gamma_{T_{n}}$ and will reach the value of $\Gamma^{\prime}=\gamma_{T_{n}}$ in order to keep the same modulation mode while reducing the average radiated power. If, on the other hand, $\Gamma \in\left[\gamma_{T_{n+1}} / G_{\max }, \gamma_{T_{n+1}}\right)$ then the HPA increases its power by $\gamma_{T_{n+1}} / \Gamma$ and will reach the value of $\Gamma^{\prime}=\gamma_{T_{n+1}}$ which allows to use the modulation mode $n+1$. The transmitter gain for continuous adaptation takes values in $\left[1 / G_{\max }, \infty\right)$.

2) Discrete Power Control: We assume in this case that the HPA's gain can only take discrete values. This gain can be adjusted using a binary feedback and a power control step size $G_{\delta}$. If $\Gamma \in\left[\gamma_{T_{n}}, \gamma_{T_{n+1}} / G_{\max }\right)$ then the HPA reduces its power by $\left\lfloor\frac{\Gamma_{\mathrm{dB}}-\gamma_{T_{n \mathrm{~dB}}}}{G_{\delta \mathrm{dB}}}\right\rfloor G_{\delta \mathrm{dB}}$, where $\lfloor\cdot\rfloor$ is the floor function. If, on the other hand, $\Gamma \in\left[\gamma_{T_{n+1}} / G_{\max }, \gamma_{T_{n+1}}\right)$ then the HPA increases its power by $\left[\frac{\gamma_{T_{n}+1 \mathrm{~dB}}-\Gamma_{\mathrm{dB}}}{G_{\delta \mathrm{dB}}}\right] G_{\delta \mathrm{dB}}$, where $\lceil\cdot 7$ is the ceil function. The transmitter gain for discrete adaptation takes $K+k$ possible values and is given by

$$
G_{\mathrm{dB}}=m G_{\delta \mathrm{dB}}, m=-k,-k+1, \ldots, 0, \ldots, K-1,(6)
$$

where

$$
k=\left\lceil\frac{\gamma_{T_{n+1 \mathrm{~dB}}}-G_{\operatorname{maxdB}}}{G_{\delta \mathrm{dB}}}\right\rceil,
$$

and the maximum power control parameter $K-1$ is restricted by an error rate constraint and is given by

$$
K-1=\min _{1 \leq n \leq N}\left\lfloor\frac{\gamma_{T n+1 \mathrm{~dB}}-G_{\operatorname{maxdB}}-\gamma_{T n \mathrm{~dB}}}{G_{\delta \mathrm{dB}}}\right\rfloor .
$$

If the HPA increases its power then the gain in $\mathrm{dB}$ is negative and will take one of the values in (6) for $m=-k,-k+$ $1, \ldots,-1$. If on the other hand we have a power decrease then 


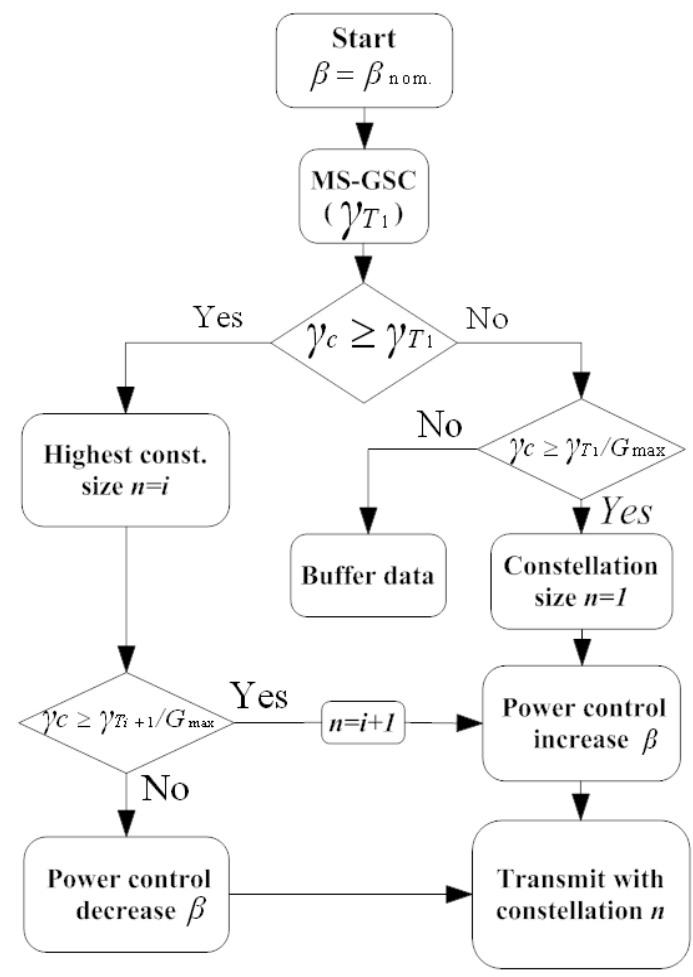

Fig. 2. Mode of operation of the JAMDCPC-1 scheme, where $\gamma_{c}$ is the combined SNR with MS-GSC $\left(\gamma_{T 1}\right)$.

we have a positive $\mathrm{dB}$ gain that is equal to $m G_{\delta \mathrm{dB}}$ where $m=1, \ldots, K-1$. The combined SNR after power control in $\mathrm{dB}, \Gamma_{\mathrm{dB}}^{\prime}$, is then equal to $\Gamma_{\mathrm{dB}}-G_{\mathrm{dB}}$ for both continuous and discrete adaptations.

\section{Mode OF Operation ANd COMbined SNR STATISTICS}

\section{A. Processing-Power Efficient JAMDCPC Scheme}

1) Mode of Operation: The mode of operation of the JAMDCPC-1 scheme is summarized in a flowchart given in Fig. 2. In the beginning of each data burst, the base station transmits a training sequence using the nominal power level $\beta_{\text {nom. }}$. After estimating and ranking the $L$ available paths, the combiner in the mobile's side tries to increase the output SNR above the threshold for the lowest constellation size by performing MS-GSC with $\gamma_{T 1}$ as output threshold. Whenever the combined SNR $\Gamma$ is larger than $\gamma_{T 1}$, the mobile stops combining and determines the highest feasible constellation index $n$ for the given $\Gamma$ by comparing the combined SNR to different switching thresholds $\left\{\gamma_{T_{n}} / G_{\max }\right\}_{n=1}^{N}$. The modulation mode $n\left(2^{n}\right.$-QAM) is selected if $\Gamma$ is greater than $\gamma_{T n}$ but strictly smaller than $\gamma_{T_{n+1}} / G_{\max }$ and, in this case, a joint power control process is initiated and the base station reduces its power level such that the modulation mode $n$ is still usable. If $\Gamma \in\left[\gamma_{T_{n+1}} / G_{\max }, \gamma_{T_{n+1}}\right)$ then the mobile asks the base station to increase its power in order to reach the constellation size $n+1$. If, even after combining all $L$ paths, the lowest constellation size is not reached (i.e. $\Gamma<\gamma_{T 1} / G_{\max }$ ), the base station will buffer the data for a channel coherence time.
In the particular case of $G_{\max }=1$, the JAMDCPC- 1 scheme reduces to the PES-JAMDC scheme proposed in [14].

2) Statistics of the Output SNR Before Power Control: The statistics of the combined SNR $\Gamma$ with the JAMDCPC-1 scheme can easily be obtained based on the mode of operation of the scheme described above. We can see that $\Gamma$ is the same as the combined SNR of MS-GSC with $\gamma_{T 1}$ as the output threshold. The cdf of the received SNR, $F_{\Gamma}(\cdot)$, of JAMDCPC1 based on MS-GSC is then given by

$$
F_{\Gamma}(\gamma)= \begin{cases}F_{\Gamma}^{\operatorname{MSC}\left(\gamma_{T 1}\right)}(\gamma), & \gamma>\gamma_{T_{1}} / G_{\max } \\ F_{\Gamma}^{\operatorname{MSC}\left(\gamma_{T_{1}}\right)}\left(\gamma_{T_{1}} / G_{\max }\right), & 0<\gamma \leq \gamma_{T_{1}} / G_{\max }\end{cases}
$$

where $F_{\Gamma}^{\mathrm{MSC}\left(\gamma_{T_{1}}\right)}(\cdot)$ denotes the cdf of the combined SNR with $L$-branch MS-GSC and using $\gamma_{T 1}$ as an output threshold. The expression of this cdf is given for the i.i.d. Rayleigh fading environment in [11, Eq. (24)].

Correspondingly, the expression of the pdf of the combined SNR with JAMDCPC- $1, f_{\Gamma}(\cdot)$, is given by

$$
\begin{aligned}
f_{\Gamma}(\gamma)= & f_{\Gamma}^{\operatorname{MSC}\left(\gamma_{T_{1}}\right)}(\gamma) \mathcal{U}\left(\gamma-\gamma_{T 1} / G_{\max }\right) \\
& +f_{\Gamma}^{\operatorname{MSC}\left(\gamma_{T 1}\right)}\left(\gamma_{T 1} / G_{\max }\right) \delta(\gamma),
\end{aligned}
$$

where $\mathcal{U}(\cdot)$ and $\delta(\cdot)$ are the unit step function and the delta function, respectively. In (10), $f_{\Gamma}^{\mathrm{MSC}\left(\gamma_{T 1}\right)}(\cdot)$ denotes the pdf of the combined SNR with $L$-branch MS-GSC and using an output threshold equal to $\gamma_{T 1}$, and which is given for the i.i.d. Rayleigh fading environment in [11, Eq. (26)].

\section{B. Bandwidth Efficient JAMDCPC Scheme}

1) Mode of Operation: This scheme is designed to maximize the spectral efficiency by: (i) performing all the the necessary diversity combining aiming for the highest signal constellation, and (ii) increasing the power level that both allows to reach the next constellation and obeys to the power constraint. The mode of operation of JAMDCPC-2 is summarized in a flowchart given in Fig. 3. In the beginning of each data burst, the base station transmits a training sequence using the nominal power level $\beta_{\text {nom. }}$. After estimating and ranking the $L$ available paths, the combiner in the mobile's side tries to increase the output SNR above the threshold for the highest constellation size by performing MS-GSC with $\gamma_{T N}$ as output threshold. Whenever the combined SNR is larger than $\gamma_{T N}$, the receiver selects the highest constellation size $(N)$ and jointly asks the transmitter to use the lowest possible power level such that the highest modulation mode $\left(2^{N}\right.$-QAM $)$ is still usable. If the combined SNR of all available branches is still below $\gamma_{T N}$, the mobile determines the highest feasible constellation size. The modulation mode $n$ is selected by the mobile if the combined SNR is strictly smaller than $\gamma_{T n+1} / G_{\max }$ but greater than $\gamma_{T_{n}}$ and a joint power control process is applied with the selected combined structure and modulation mode $n$. If even the lowest constellation size is not feasible, data are buffered for a channel coherence time. In the particular case of $G_{\max }=1$, the JAMDCPC-2 scheme reduces to the BES-JAMDC scheme proposed in [14].

2) Statistics of the Output SNR Before Power Control: The statistics of the combined SNR $\Gamma$ with JAMDCPC-2 can easily be obtained based on the mode of operation of the scheme described above. We can see that $\Gamma$ is the same as the 


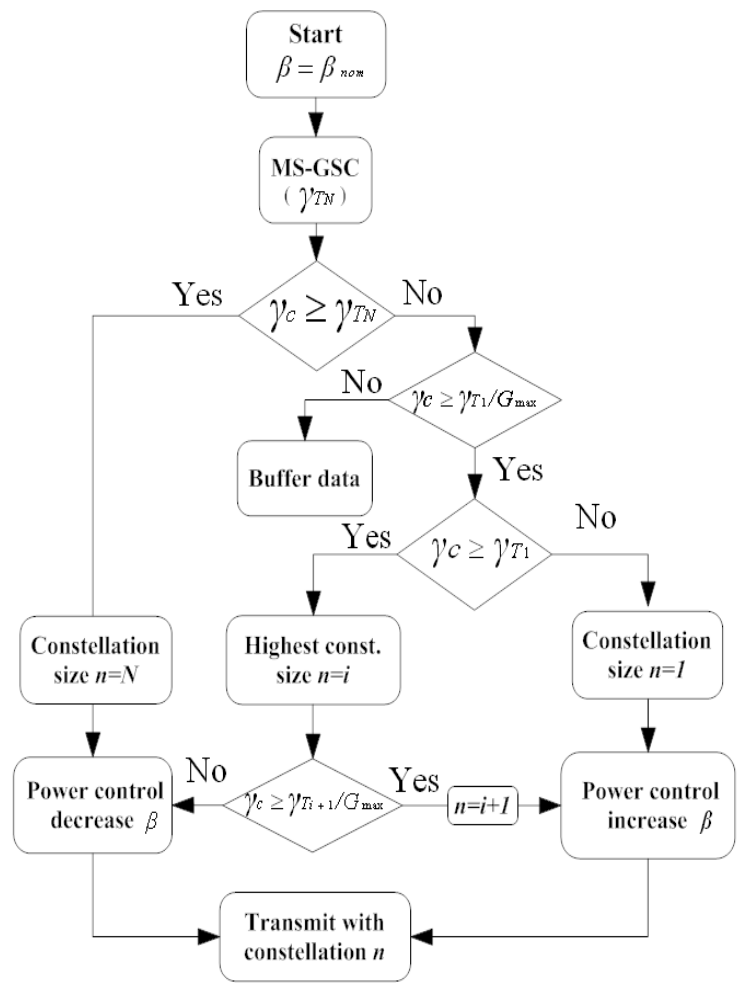

Fig. 3. Mode of operation of the JAMDCPC-2 scheme, where $\gamma_{c}$ is the combined SNR with MS-GSC $\left(\gamma_{T N}\right)$.

combined SNR of MS-GSC with $\gamma_{T N}$ as the output threshold. The cdf of the received SNR, $F_{\Gamma}(\cdot)$, of JAMDCPC-2 based on MS-GSC is given by

$$
F_{\Gamma}(\gamma)= \begin{cases}F_{\Gamma}^{\operatorname{MSC}\left(\gamma_{T N}\right)}(\gamma), & \gamma>\gamma_{T_{1}} / G_{\max } \\ F_{\Gamma}^{\operatorname{MSC}\left(\gamma_{T N}\right)}\left(\gamma_{T_{1}} / G_{\max }\right), & 0<\gamma \leq \gamma_{T_{1}} / G_{\max }\end{cases}
$$

Correspondingly, the expression of the pdf of the combined SNR with JAMDCPC- $2, f_{\Gamma}(\cdot)$, is given by

$$
\begin{aligned}
f_{\Gamma}(\gamma)= & f_{\Gamma}^{\operatorname{MSC}\left(\gamma_{T_{N}}\right)}(\gamma) \mathcal{U}\left(\gamma-\gamma_{T_{N}} / G_{\max }\right) \\
& +f_{\Gamma}^{\operatorname{MSC}\left(\gamma_{T_{N}}\right)}\left(\gamma_{T_{1}} / G_{\max }\right) \delta(\gamma) .
\end{aligned}
$$

\section{Bandwidth Efficient with Finger Deactivation JAMDCPC Scheme}

1) Mode of Operation: The combiner with JAMDCPC-3 start by performing MS-GSC with $\gamma_{T N}$ as output threshold. Whenever the combined SNR is larger than $\gamma_{T N}$, the receiver selects the highest constellation size $(N)$ and jointly asks the transmitter to use the lowest possible power level such that the highest modulation mode $\left(2^{N}\right.$-QAM) is still usable. If the modulation mode $n$ is selected by the mobile and the combined SNR is strictly smaller than $\gamma_{T n+1} / G_{\max }$ but greater than $\gamma_{T_{n}}$, the JAMDCPC-3 scheme initiates a joint finger deactivation and power control processes. During the finger deactivation process the mobile selects the minimum number of paths that are needed such that the output SNR remains greater than $\gamma_{T_{n}}$ (i.e. turning off the weakest branches while conserving the same modulation mode). The deactivation process is continued until turning off another diversity path leads to an output SNR below $\gamma_{T_{n}}$. After this, the base station reduces its power level such that the selected constellation is still usable. If on the other hand $\Gamma \in\left[\gamma_{T_{n+1}} / G_{\max }, \gamma_{T_{n+1}}\right)$ then the receiver jointly selects the current combiner structure, the modulation mode $n+1$, and the needed power control increase level. If even the lowest constellation size is not feasible, data are buffered for a channel coherence time. In the particular case of $G_{\max }=1$, the JAMDCPC-3 scheme reduces to the bandwidth efficient and power greedy scheme (BES-FD) proposed in [12].

2) Statistics of the Output SNR Before Power Control: Based on the mode of operation described above and using the expression of the cdf of the combined SNR of the bandwidth efficient and power greedy scheme proposed in [12], we give the expression of the cdf of the combined SNR before power control of the JAMDCPC-3 scheme as

$F_{\Gamma}(x)=$

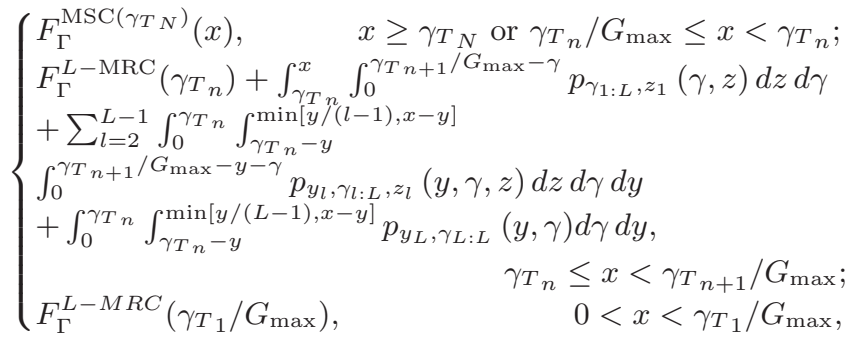

where $\gamma_{l: L}$ denotes the SNR of the $l$-th strongest path and $F_{\Gamma}^{L-M R C}(\cdot)$ is the cdf of the combined SNR with $L$-branch MRC scheme and is given in closed form for i.i.d. Rayleigh fading in $[9$, Eq. (25)] by

$$
F_{\Gamma}^{L-\operatorname{MRC}}(x)=1-e^{-(x / \bar{\gamma})} \sum_{l=0}^{L-1} \frac{\left(\frac{x}{\bar{\gamma}}\right)^{l}}{l !} .
$$

The closed-form expressions of the joint pdfs $p_{\gamma_{1: L}, z_{1}}(\gamma, z)$ and $p_{y_{L}, \gamma_{L: L}}(y, \gamma)$ can be easily obtained as marginals of the joint pdf $p_{y_{l}, \gamma_{l: L}, z_{l}}(y, \gamma, z)$ given in closed-form for i.i.d. Rayleigh fading in [16, Eq. (20)] by

$p_{y_{l}, \gamma_{l: L}, z_{l}}(y, \gamma, z)=$
$\frac{L !}{(L-1) !(l-1) \bar{\gamma}^{L}} \frac{[y-(l-1) \gamma]^{l-2}}{(l-2) !(L-l-1) !} e^{-\frac{y+\gamma+z}{\gamma}} \mathcal{U}(y-(l-1) \gamma)$

$\times \sum_{i=0}^{L-l}\left(\begin{array}{c}L-l \\ i\end{array}\right)(-1)^{i}(z-i \gamma)^{L-l-1} \mathcal{U}(z-i \gamma)$,

$\gamma>0, y>(l-1) \gamma, z<(L-l) \gamma$.

After differentiating $F_{\Gamma}(x)$ with respect to $x$, a generic formula for the pdf of the output SNR with JAMDCPC-3 can be found as

$f_{\Gamma}(x)=$

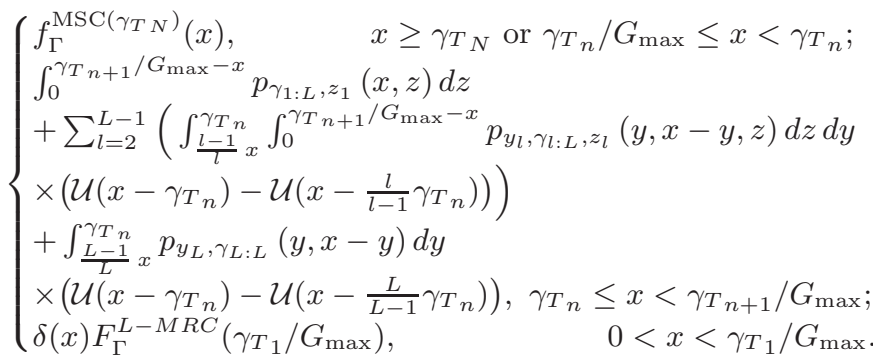




\section{PERformance AnAlysis}

\section{A. Transmitted Power Gain}

While in the JAMDC schemes, the transmitter starts sending using its maximal power, in the proposed JAMDCPC schemes the transmitter starts sending using its nominal power. Based on this nominal initial power transmitted and on the MS-GSC diversity combining, we assume that we have a combined SNR of $\Gamma$ and that we reached the constellation size $n$. The transmitter will then vary its power depending on the value of $\Gamma$.

1) Continuous Power Control: The case of continuous power adaptation will allow us to reach exactly the value of $\gamma_{T_{n}}$. If the combined SNR before power control $\Gamma$ verifies $\gamma_{T n} / G_{\max }<\Gamma \leq \gamma_{T n}$ then transmitter will increase its power by $\gamma_{T_{n}} / \Gamma$. If on the other hand $\gamma_{T_{n}}<\Gamma \leq$ $\gamma_{T_{n+1}} / G_{\max }$, the transmitter will reduce its power by $\Gamma / \gamma_{T_{n}}$. In both these cases the transmitted power gain is given by $G=\Gamma / \gamma_{T_{n}}$. Hence, the average transmitted power gain, in decibels, $\bar{G}_{\mathrm{dB}}$, is given by

$$
\begin{aligned}
& \bar{G}_{\mathrm{dB}}=\sum_{n=1}^{N} \int_{\gamma_{T n} / G_{\max }}^{\gamma_{T n+1} / G_{\max }} 10 \log _{10}\left(\frac{\Gamma}{\gamma_{T_{n}}}\right) f_{\Gamma}(\Gamma) d \Gamma \\
& =\sum_{n=1}^{N} \int_{\gamma_{T n} / G_{\max }}^{\gamma_{T n+1} / G_{\max }} 10 \log _{10}(\Gamma) f_{\Gamma}(\Gamma) d \Gamma \\
& -\sum_{n=1}^{N} 10 \log _{10}\left(\gamma_{T_{n}}\right)\left[F_{\Gamma}\left(\gamma_{T_{n+1}} / G_{\max }\right)-F_{\Gamma}\left(\gamma_{T_{n}} / G_{\max }\right)\right]
\end{aligned}
$$

where we define $\gamma_{T N+1}=\infty$.

2) Discrete Power Control: Let us start by summarizing the choice of the power parameter $\beta$ for the discrete power adaptation. For $\gamma_{T n} / G_{\max }<\Gamma \leq \gamma_{T n+1} / G_{\max }, n \geq 1$, and $-k \leq m \leq K-1$

$$
\begin{aligned}
& \beta=\beta_{K-1}, \quad \text { iff } \quad \frac{\Gamma}{\gamma_{T_{n}}} \geq \beta_{K-1} \\
& \beta=\beta_{m-1}, \quad \text { iff } \quad \beta_{m-1} \leq \frac{\Gamma}{\gamma_{T_{n}}}<\beta_{m} \\
& \beta=\beta_{0}=1, \quad \text { iff } \quad \beta_{0} \leq \frac{\Gamma}{\gamma_{T_{n}}}<\beta_{1} \\
& \beta=\beta_{-m+1}, \quad \text { iff } \quad \beta_{-m+1} \leq \frac{\Gamma}{\gamma_{T_{n}}}<\beta_{-m} \\
& \beta=\beta_{-k}=\frac{1}{G_{\max }} \text {, iff } \quad \beta_{-k} \leq \frac{\Gamma}{\gamma_{T_{n}}}<\beta_{-k+1}
\end{aligned}
$$

The average transmitted $\mathrm{dB}$ power gain is then given by

$$
\begin{aligned}
\bar{G}_{\mathrm{dB}} & =\sum_{n=1}^{N} \int_{\gamma_{T n} / G_{\max }}^{\gamma_{T n+1} / G_{\max }} 10 \log _{10}\left(\frac{\Gamma}{\Gamma / \beta}\right) f_{\Gamma}(\Gamma) d \Gamma \\
& =\sum_{n=1}^{N} \int_{\gamma_{T n} / G_{\max }}^{\gamma_{T n+1} / G_{\max }} 10 \log _{10}(\beta) f_{\Gamma}(\Gamma) d \Gamma \\
& =\sum_{n=1}^{N} \sum_{m=-k}^{K-1} 10 \log _{10}\left(\beta_{m}\right)\left(F_{\Gamma}\left(\gamma_{T_{n}} \beta_{m+1}\right)-F_{\Gamma}\left(\gamma_{T_{n}} \beta_{m}\right)\right),
\end{aligned}
$$

where $\gamma_{T_{n}} \beta_{K}=\gamma_{T_{n+1}} / G_{\max }$.

\section{B. Average Number of Combined Paths}

In this paper, we quantify the power consumption for diversity combining in terms of the average number of combined paths and we do not consider the processing load needed to run the proposed schemes.

For the JAMDCPC-1 scheme, it can be shown that the average number of combined paths is given by

$$
\bar{N}_{c}=1+\sum_{i=1}^{L-1} F_{\Gamma}^{L / i-\mathrm{GSC}}\left(\gamma_{T_{1}}\right)-L F_{\Gamma}^{L-\mathrm{MRC}}\left(\gamma_{T_{1}} / G_{\max }\right),
$$

where $F_{\Gamma}^{L / i-\mathrm{GSC}}(\cdot)$ is the cdf of the combined SNR with $L / i$ $\mathrm{GSC}^{3}$ scheme and is given in closed-form for i.i.d. Rayleigh fading in [17] by

$$
\begin{aligned}
F_{\Gamma}^{L / i-\mathrm{GSC}}(\gamma)= & 1-\sum_{l=0}^{i-1} \frac{A_{l} \gamma^{l} e^{-\gamma / \bar{\gamma}}}{\bar{\gamma}^{l} l !} \\
& +\sum_{k=1}^{L-i} B_{k} e^{-(1+k / i) \gamma / \bar{\gamma}}, \quad \gamma \geq 0,
\end{aligned}
$$

where

$$
\begin{gathered}
A_{l}=\sum_{j=0}^{i-l-1} a_{l+j}=a_{l}+A_{l+1}, \text { with } A_{-1}=0 \\
a_{i-1-l}=\left(\begin{array}{c}
L \\
i
\end{array}\right) \sum_{k=1}^{L-i}\left(\begin{array}{c}
L-i \\
k
\end{array}\right) \frac{(-1)^{k+l+1} i^{l}}{k^{l}} \\
B_{k}=\frac{i b_{k}}{k+i}, \\
b_{k}=\left(\begin{array}{c}
L \\
i
\end{array}\right)\left(\begin{array}{c}
L-i \\
k
\end{array}\right) \frac{(-1)^{i+k} i^{i-1}}{k^{i-1}} .
\end{gathered}
$$

Using (14) and (21), we obtain the average number of combined paths of the JAMDCPC-1 scheme in closed form as

$$
\begin{aligned}
\bar{N}_{c}= & \sum_{i=1}^{L-1}\left[\sum_{k=1}^{L-i} B_{k} e^{-(1+k / i) \gamma_{T 1} / \bar{\gamma}}-\sum_{l=0}^{i-1} \frac{A_{l} \gamma_{T 1}{ }^{l} e^{-\gamma_{T 1} / \bar{\gamma}}}{\bar{\gamma}^{l} l !}\right] \\
& +L e^{-\frac{\gamma_{T 1}}{\bar{\gamma} G_{\max }}} \sum_{l=0}^{L-1} \frac{\left(\frac{\gamma_{T 1}}{\bar{\gamma} G_{\max }}\right)^{l}}{l !}
\end{aligned}
$$

Similarly, it can be shown that the average number of combined paths for the JAMDCPC-2 scheme is given by

$$
\bar{N}_{c}=1+\sum_{i=1}^{L-1} F_{\Gamma}^{L / i-\mathrm{GSC}}\left(\gamma_{T_{N}}\right)-L F_{\Gamma}^{L-\mathrm{MRC}}\left(\gamma_{T_{1}} / G_{\max }\right)
$$

which is also given in closed form by

$$
\begin{aligned}
\bar{N}_{c}= & \sum_{i=1}^{L-1}\left[\sum_{k=1}^{L-i} B_{k} e^{-(1+k / i) \gamma_{T N} / \bar{\gamma}}-\sum_{l=0}^{i-1} \frac{A_{l} \gamma_{T N}{ }^{l} e^{-\gamma_{T N} / \bar{\gamma}}}{\bar{\gamma}^{l} l !}\right] \\
& +L e^{-\frac{\gamma_{T 1}}{\bar{\gamma} G_{\max }}} \sum_{l=0}^{L-1} \frac{\left(\frac{\gamma_{T 1}}{\bar{\gamma} G_{\max }}\right)^{l}}{l !}
\end{aligned}
$$

${ }^{3} L / i$-GSC adaptively combines the $i$ strongest resolvable paths among the $L$ available ones [7]-[9]. 
The average number of combined paths for the JAMDCPC-3 can be calculated as [12, Eq. (24), Option 2]

$$
\overline{N_{c}}=\sum_{l=1}^{L} \sum_{n=1}^{N} l P_{l, n},
$$

where $P_{l, n}$ denotes the probability that mode $n$ is used with $l$ combined branches.

Based on the mode of operation of the JAMDCPC-3, the expression of $P_{l, n}$ is given, for $n=N$, by

$$
\begin{aligned}
& P_{l, N} \\
& = \begin{cases}1-F_{\Gamma}^{L / 1-\mathrm{GSC}}\left(\gamma_{T N} / G_{\max }\right), & l=1 ; \\
F_{\Gamma}^{L /(l-1)-\mathrm{GSC}}\left(\gamma_{T N} / G_{\max }\right) \\
-F_{\Gamma}^{L / l-\mathrm{GSC}}\left(\gamma_{T N} / G_{\max }\right), & 1<l \leq L ;\end{cases}
\end{aligned}
$$

and for $n<N$ by

$P_{l, n}$

$$
=\left\{\begin{array}{lc}
\operatorname{Pr}\left[\gamma_{T_{n}} \leq \gamma_{1: L} \& \sum_{j=1}^{L} \gamma_{j: L}<\gamma_{T_{n+1}} / G_{\max }\right], & l=1 ; \\
\operatorname{Pr}\left[\sum_{j=1}^{l-1} \gamma_{j: L}<\gamma_{T_{n}} \leq \sum_{j=1}^{l} \gamma_{j: L}\right. & 1<l<L ; \\
\left.\& \sum_{j=1}^{L} \gamma_{j: L}<\gamma_{T_{n+1}} / G_{\max }\right], & \left.l<\gamma_{j: L}<\gamma_{T_{n+1}} / G_{\max }\right] \\
\operatorname{Pr}\left[\sum_{j=1}^{L-1} \gamma_{j: L}<\gamma_{T_{n}} \leq \sum_{j=1}^{L} \gamma_{j: L}\right. & l=L ;
\end{array}\right.
$$

Using the same steps and the definitions of the joint pdf's of $y_{l}, \gamma_{l: L}$, and $z_{l}$ as in [12], and taking $G_{\max }$ into consideration, (31) can be calculated as

$$
\begin{aligned}
& P_{l, n}
\end{aligned}
$$

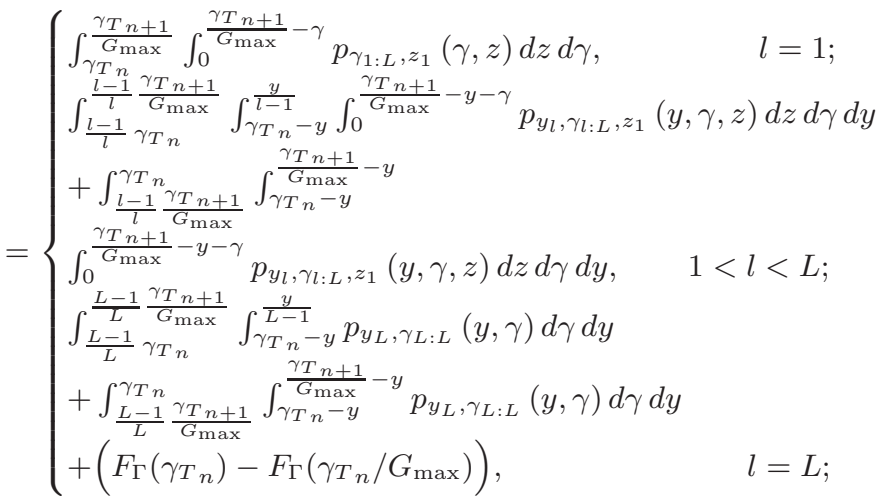

where $F_{\Gamma}(\cdot)$ is the cdf of the combined SNR with JAMDCPC3 and it is given in (13).

\section{Average Spectral Efficiency}

A general expression of the average spectral efficiency of an adaptive modulation system is given in [15, Eq. (33)] by

$$
\eta=\sum_{i=1}^{N} n p_{n}
$$

where $p_{n}$ denotes the probability that the $n$th constellation is used. The expression of this probability is given for the JAMDCPC-1 scheme by

$$
p_{n}=F_{\Gamma}^{\mathrm{MSC}\left(\gamma_{T 1}\right)}\left(\gamma_{T_{n+1}} / G_{\max }\right)-F_{\Gamma}^{\mathrm{MSC}\left(\gamma_{T 1}\right)}\left(\gamma_{T_{n}} / G_{\max }\right) .
$$

Using the above expression of $p_{n}$ and (33) we obtain the following expression of the average spectral efficiency of the JAMDCPC-1 scheme

$$
\eta=N-\sum_{n=1}^{N} F_{\Gamma}^{\mathrm{MSC}\left(\gamma_{T_{1}}\right)}\left(\gamma_{T_{n}} / G_{\max }\right) .
$$

Similarly, the expression of $p_{n}$ for JAMDCPC-2, which is equal to that of JAMDCPC-3, can be shown to be given by

$$
p_{n}=F_{\Gamma}^{\mathrm{MSC}\left(\gamma_{T N}\right)}\left(\gamma_{T_{n+1}} / G_{\max }\right)-F_{\Gamma}^{\mathrm{MSC}\left(\gamma_{T_{N}}\right)}\left(\gamma_{T_{n}} / G_{\max }\right) .
$$

Then JAMDCPC-2 and JAMDCPC-3 have the same spectral efficiency given by

$$
\eta=N-\sum_{n=1}^{N} F_{\Gamma}^{\mathrm{MSC}\left(\gamma_{T_{N}}\right)}\left(\gamma_{T_{n}} / G_{\max }\right) .
$$

\section{Statistics of the Output SNR After Power Control}

In order to analyze the bit error rate performance of the three proposed schemes, a statistical characterization of the combined SNR after power control is needed. Based on the mode of operation of the JAMDCPC schemes, that we described in Section II, we give in what follows the expressions of the pdf and the cdf of the combined SNR after power control for both continuous and discrete adaptations.

1) Continuous Power Control: Assuming that the modulation mode $n$ has been chosen, the combined SNR after con(32) tinuous power control, $\Gamma^{\prime}$, will be set to $\gamma_{T_{n}}$. The expression of the PMF is then given, for $0 \leq n \leq N$, by

$$
\begin{aligned}
& f_{\Gamma^{\prime}}\left(\Gamma^{\prime}\right) \\
& = \begin{cases}F_{\Gamma}\left(\gamma_{T_{n+1}} / G_{\max }\right)-F_{\Gamma}\left(\gamma_{T_{n}} / G_{\max }\right), & \Gamma^{\prime}=\gamma_{T_{n}} ; \\
0, & \text { otherwise; }\end{cases}
\end{aligned}
$$

where we define $\gamma_{T_{0}}=0$, and $\gamma_{T N+1}=\infty$.

The expression of the cdf is then given, for $0 \leq n \leq N$, by

$$
\begin{aligned}
& F_{\Gamma^{\prime}}\left(\Gamma^{\prime}\right) \\
& = \begin{cases}F_{\Gamma}\left(\gamma_{T_{n+1}} / G_{\max }\right), & \gamma_{T_{n}} / G_{\max } \leq \Gamma^{\prime}<\gamma_{T_{n+1}} / G_{\max } ; \\
0, & \Gamma^{\prime}<0\end{cases}
\end{aligned}
$$

2) Discrete Power Control: If the combined SNR before power control falls between $\gamma_{T n}$ and $\gamma_{T n+1}$ the transmitter will vary its power in order to use the constellation size $n$ or $n+1$ with a minimum amount of transmitted power. Starting from the mode of operation of the proposed schemes, using the constraint on the length of $\left[\gamma_{T_{n}}, \gamma_{T_{n+1}} / G_{\max }\right]$ given by (8), and using the same steps as in [14], we give a simplified expression for the pdf of the combined SNR after power control as

$f_{\Gamma^{\prime}}\left(\Gamma^{\prime}\right)$

$$
=\left\{\begin{array}{lr}
\sum_{j=-k}^{K-2} \beta_{j} f_{\Gamma}\left(\beta_{j} \Gamma^{\prime}\right) & \gamma_{T_{n}} \leq \Gamma^{\prime}<\gamma_{T_{n}} \beta_{1} ; \\
+\beta_{K-1} f_{\Gamma}\left(\beta_{K-1} \Gamma^{\prime}\right) \mathcal{U}\left(\frac{\gamma_{T n+1}}{\beta_{K-1} G_{\max }}-\Gamma^{\prime}\right), & \\
\beta_{K-1} f_{\Gamma}\left(\beta_{K-1} \Gamma^{\prime}\right), & \\
\quad \gamma_{T_{n}} \beta_{1} \leq \Gamma^{\prime}<\frac{\gamma_{T n+1}}{\beta_{K-1} G_{\max }} \& \gamma_{T_{n}} \beta_{K-1} G_{\delta} \leq \frac{\gamma_{T n+1}}{G_{\max }} ; \\
0, & \text { otherwise; }
\end{array}\right.
$$




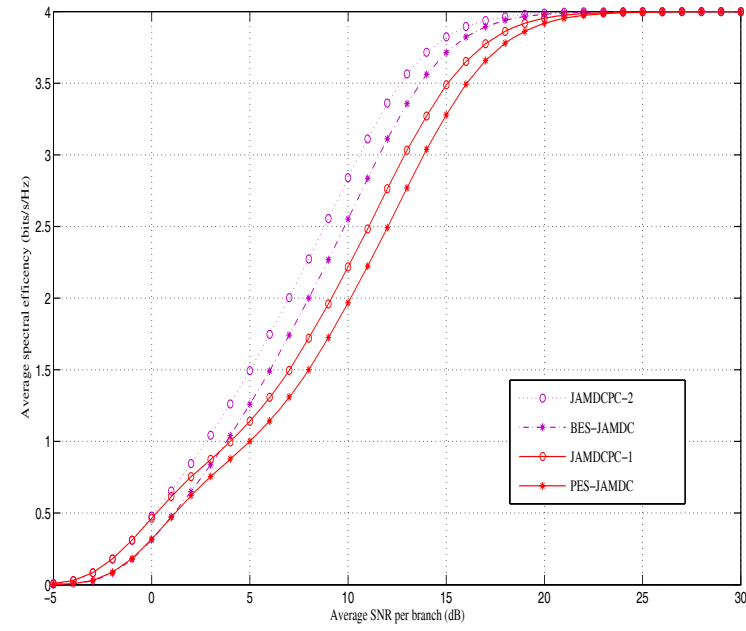

Fig. 4. Average spectral efficiency versus the average SNR per branch, $\bar{\gamma}$, comparison between the JAMDC and JAMDCPC schemes.

\section{E. Average Error Rate}

The general expression of the average BER for an adaptive modulation system is given in [15, Eq. (35)] as

$$
\overline{P_{b}}=\frac{1}{\eta} \sum_{n=1}^{N} n \overline{P_{b_{n}}}
$$

where $\overline{P_{b_{n}}}$ is the average BER for constellation size $n$, and is given, using (4), by

$$
\overline{P_{b_{n}}}=\int_{\gamma_{T_{n}} / G_{\max }}^{\gamma_{T_{n+1}} / G_{\max }} P_{b_{n}}\left(\Gamma^{\prime}\right) f_{\Gamma^{\prime}}\left(\Gamma^{\prime}\right) d \Gamma^{\prime} .
$$

\section{NUMERICAL EXAMPLES}

The performance results studied above were double checked for their accuracy via Monte Carlo simulations. The performance of the JAMDCPC schemes is illustrated in this section with some selected numerical results. For these examples we set the number of available diversity branches $L=3$, the number of signal constellations $N=4$, the maximum value of the $\mathrm{dB}$ additional gain $G_{\operatorname{maxdB}}=1 \mathrm{~dB}$, and the bit error rate constraint as $P_{b_{0}}=10^{-3}$.

In the particular case of $G_{\max }=1$ (i.e. $G_{\operatorname{maxdB}}=0$ ), the performance of our proposed schemes will reduce to that of the JAMDC schemes that we use in this section as comparison with the JAMDCPC schemes.

\section{A. Average Spectral Efficiency and Number of Combined Paths}

Fig. 4 illustrates the spectral efficiency improvement that is offered by the the proposed JAMDCPC schemes over the JAMDC schemes. This improvement comes at the expense of a higher number of combined paths in the low SNR range as shown in Fig. 5. These results are explained by the fact that the transmitter in the JAMDC schemes used to buffer the data whenever the combined SNR does not reach the lowest constellation size after combining all the available $L$ paths,

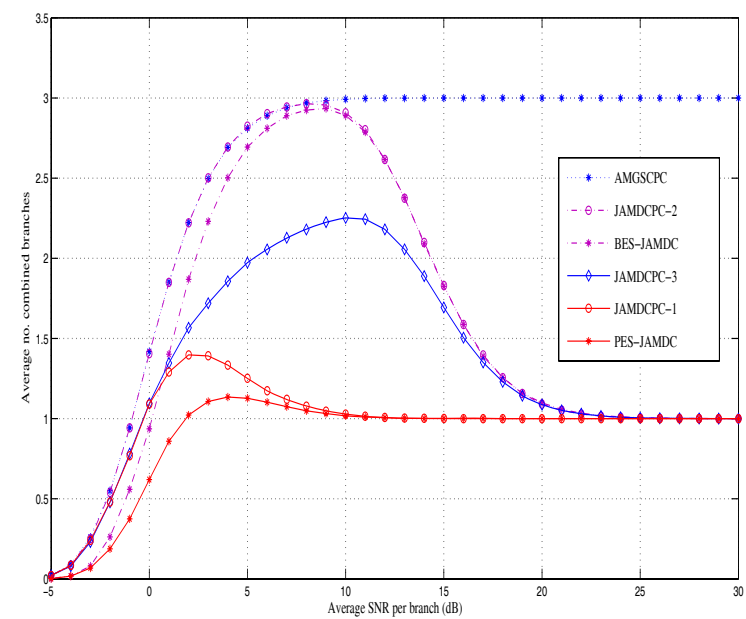

Fig. 5. Average number of combined paths versus the average SNR per branch, $\bar{\gamma}$, comparison between the JAMDC and JAMDCPC schemes.

but in the JAMDCPC schemes if the combined SNR $\Gamma$ is lower than $\gamma_{T 1}$ but higher than $\gamma_{T_{1}} / G_{\max }$, the transmitter will send using the lowest constellation size and combining all the $L$ available paths. For an average SNR above $20 \mathrm{~dB}$, we can see that for both JAMDC and JAMDCPC schemes one diversity path is enough to use the highest constellation size (i.e. 16-QAM modulation).

We can also see from Fig. 5 that, thanks to the finger deactivation process, the JAMDCPC-3 scheme has better processing power performance than JAMDCPC-2 while keeping the same spectral efficiency.

For reference we give the average number of combined branches for a bandwidth efficient scheme using adaptive modulation, GSC, and power control (AMGSCPC). For the low SNR range AMGSCPC needs as much processing power as our bandwidth efficient scheme but in the high SNR range all our schemes combine only one branch while AMGSCPC always combines all the available paths. This processing power performance improvement in our schemes comes with a slightly higher computational complexity resulting from comparing the combined SNR to the MS-GSC switching threshold after each combination of a new available path.

\section{B. Transmitted Power Gain}

Fig. 6 compares the average transmitted power gain of our three proposed schemes. We can clearly see from this figure that the JAMDCPC-3 scheme has the lowest average transmitted $\mathrm{dB}$ gain. This is explained by the fact that the finger deactivation process will turn off all the weakest branches while conserving the same modulation mode. We can also see from this figure that for the low SNR range the JAMDCPC-2 scheme has higher average transmitted power gain than the JAMDCPC-1, or equivalently lower average radiated power, since the processing power efficient scheme will stop combining as soon as $\Gamma \geq \gamma_{T 1}$ while the JAMDCPC2 continue the combining process allowing to reach higher SNR and higher power gain. 


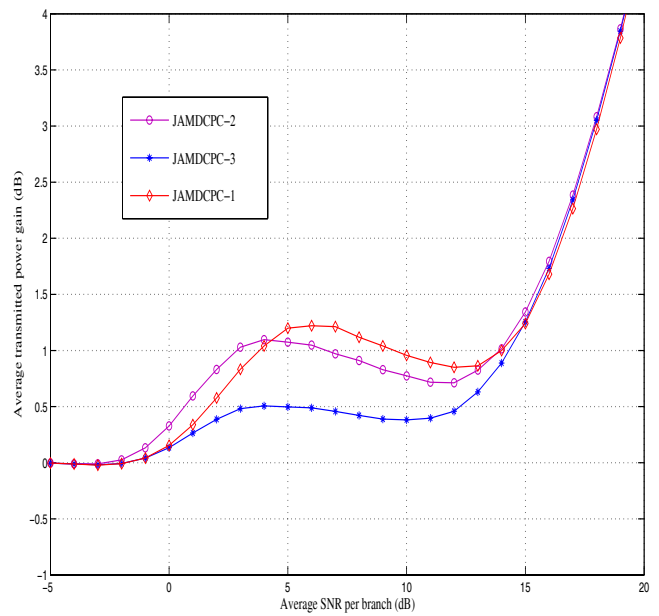

Fig. 6. Average transmitted power for continuous adaptation versus the average SNR per branch, $\bar{\gamma}$, for the three proposed schemes.

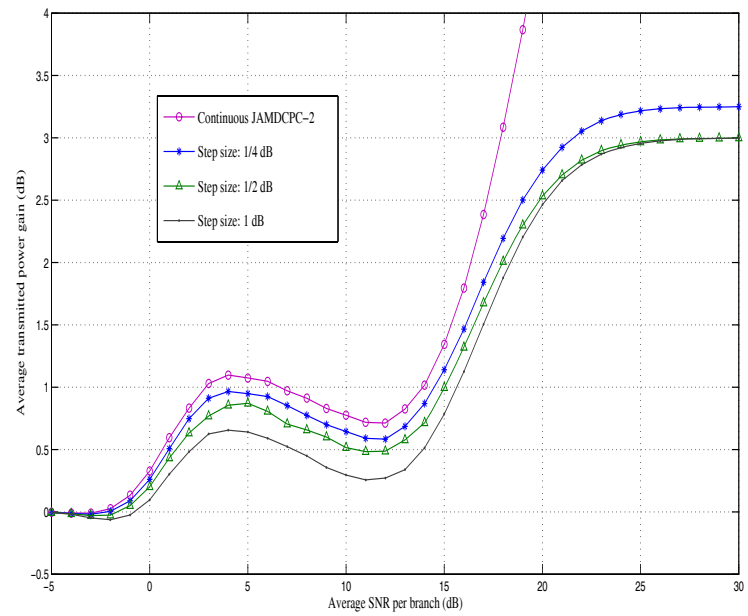

Fig. 7. Average transmitted power for the JAMDCPC-2 scheme versus the average SNR per branch, $\bar{\gamma}$.

In Fig. 7, we depict the average transmitted power gain versus the average SNR per branch for the JAMDCPC-2 scheme for both, continuous and discrete adaptations. This figure confirms that the introduction of power control reduces significantly the average radiated power. We also see from this figure that this lower average radiated power is also obtained by reducing the power control step size which increases the power gain. According to the expression of $K-1$ given in (8), the maximum reduction for discrete level transmitted power control is limited by the length of the shortest interval. This explains why the average transmitted power gain saturates in different values depending on the used power control step size. This is not the case for the continuous power adaptation since for the high SNR range the gain with continuous power control is unbounded.

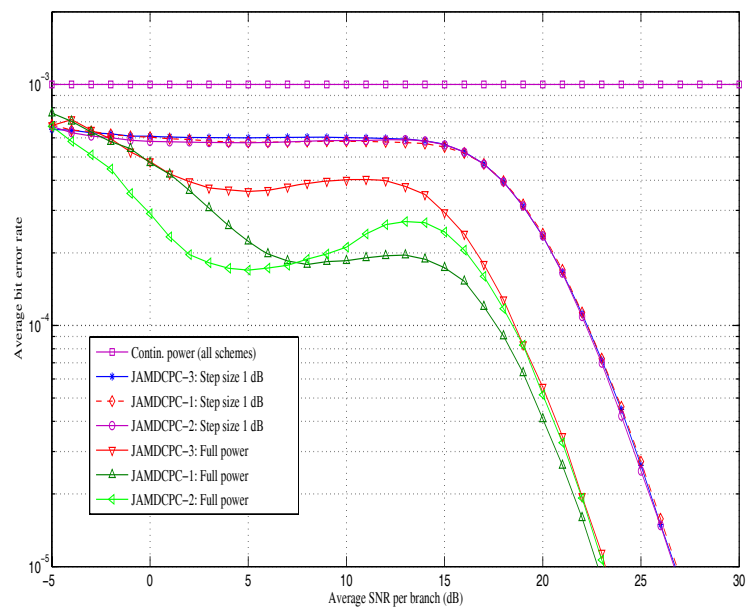

Fig. 8. Average bit error rate versus the average SNR per branch, $\bar{\gamma}$, when $L=3, N=4$, and with a BER constraint $P_{b_{0}}=10^{-3}$.

\section{BER Performance}

In Fig. 8, we show the BER of the proposed schemes. For continuous power control adaptation, the three schemes have the same BER performance. For this case, the BER is constant and is equal to $P_{b_{0}}$, since the combined SNR after continuous power control will be set to the switching threshold corresponding to the used constellation. For discrete power control adaptation, we show that the JAMDCPC- 2 scheme has slightly better error performance than the JAMDCPC-1 scheme. The reason behind this is that in the low SNR range the JAMDCPC-2 scheme needs to combine more branches than the JAMDCPC-1. We also show in this figure that the JAMDCPC-3 has a slightly higher BER than the two other schemes. The reason behind this is the finger deactivation process which decreases the average number of combined paths while keeping a higher average transmitted power. For reference, we also compare the BER performances of JAMDCPC-2, JAMDCPC-1, and JAMDCPC-3 schemes using constant full power.

\section{CONCLUSION}

We have proposed and analyzed in this paper, three new schemes using a joint adaptive modulation, diversity combining, and downlink power control. These schemes can be viewed as general variants of some existent joint adaptive modulation and diversity combining schemes by the introduction of a common joint power control process that can both increase and decrease the power level. The JAMDCPC2 scheme has higher spectral efficiency that the proposed processing power efficient scheme. This better performance comes at the expense of a larger number of combined branches. The JAMDCPC-3 scheme was proposed in order to reduce the processing power consumption of JAMDCPC1 while keeping the spectral efficiency as high as the second scheme. Compared to existing schemes, our proposed schemes have better performance with a slight increase in the computational complexity explained by the large number of operations 
performed by the receiver during the guard periods. For a fair comparison, energy and spectrum resources used by these operations need to be taken into account.

\section{REFERENCES}

[1] J. Cavers, "Variable-rate transmission for Rayleigh fading channels," IEEE Trans. Commun., vol. 20, no. 1, pp. 15-22, Feb. 1972.

[2] A. Svensson, "An introduction to adaptive QAM modulation schemes for known and predicted channels," Proc. IEEE, vol. 95, no. 12, pp. 2322-2336, Dec. 2007.

[3] D. I. Kim, "Adaptive selection/maximal-ratio combining based on error detection of multidimensional multicode DS-CDMA," IEEE Trans. Commun., vol. 52, no. 3, pp. 446-456, Feb. 2004.

[4] A. Lioumpas, G. Karagiannidis, and T. Tsiftsis, "Adaptive generalized selection combining (A-GSC) receivers," IEEE Trans. Wireless Commun., vol. 7, no. 12, pp. 5214-5219, Dec. 2008.

[5] J. Zander, "Performance of optimum transmitter power control in cellular radio systems," IEEE Trans. Veh. Technol., vol. 41, no. 1, pp. 57-62, Feb. 1992.

[6] T.-H. Lee, J.-C. Lin, and Y. Su, "Downlink power control algorithms for cellular radio systems," IEEE Trans. Veh. Technol., vol. 44, no. 1, pp. 89-94, Feb. 1995.

[7] N. Kong, T. Eng, and L. B. Milstein, "A selection combining scheme for RAKE receivers," in Proc. IEEE Int. Conf. Univ. Personal Commun. (ICUPC'95), Tokyo, Japan, Nov. 1995, pp. 426-429.

[8] M. Z. Win and Z. A. Kostic, "Virtual path analysis of selective RAKE receiver in dense multipath channels," IEEE Commun. Lett., vol. 3, pp. 308-310, Nov. 1999

[9] M.-S. Alouini and M. K. Simon, "An MGF-based performance analysis of generalized selection combining over Rayleigh fading channels," IEEE Trans. Commun., vol. 48, no. 3, pp. 401-415, Mar. 2000.

[10] S. W. Kim, D. S. Ha, and J. H. Reed, "Minimum selection GSC and adaptive low-power RAKE combining scheme," in Proc. IEEE Int. Symp. Circuit Syst. (ISCAS'03), Bangkok, Thailand, May 2003, pp. 357360.

[11] H.-C. Yang, "New results on ordered statistics and analysis of minimum selection generalized selection combining (GSC)," IEEE Trans. Wireless Commun., vol. 5, no. 7, pp. 1876-1885, July 2006.

[12] H.-C. Yang, N. Belhaj, and M.-S. Alouini, "Performance analysis of joint adaptive modulation and diversity combining over fading channels," IEEE Trans. Commun., vol. 55, no. 3, pp. 520-528, Mar. 2007.

[13] Z. Bouida, N. Belhaj, M.-S. Alouini, and K. Qaraqe, "Minimum selection GSC with down-link power control," IEEE Trans. Wireless Commun., vol. 7, no. 7, pp. 2492-2501, July 2008.

[14] A. Gjendemsjø, H. C. Yang, G. E. Øien, and M.-S. Alouini, "Minimum selection GSC with adaptive modulation and post-combining power control," in Proc. IEEE Wireless Commun. Netw. Conf. (WCNC'2007), Hong Kong, China, Mar. 2007, pp. 2006-2011.

[15] M.-S. Alouini and A. J. Goldsmith, "Adaptive modulation over Nakagami fading channels," Wireless Pers. Commun., vol. 13, pp. 119-143, May 2000.

[16] Y.-C. Ko, H.-C. Yang, S.-S. Eom, and M.-S. Alouini, "Adaptive modulation with diversity combining based on output-threshold MRC," IEEE Trans. Wireless Commun., vol. 6, no. 10, pp. 3728-3737, Oct. 2007.
[17] Y. Roy, J.-Y. Chouinard, and S. Mahmoud, "Selection diversity combining with multiple antennas for mm-wave indoor wireless channels," IEEE J. Sel. Areas Commun., vol. 14, pp. 674-682, May 1996.

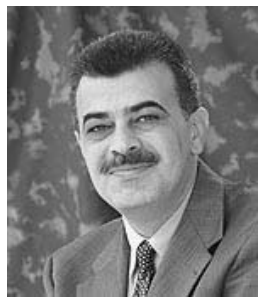

Khalid A. Qaraqe (M'97, S'00) was born in Bethlehem. He received the B.S. degree in EE from the University of Technology, Baghdad in 1986, with honors. He received the M.S. degree in EE from the University of Jordan, Jordan, in 1989, and he earned his $\mathrm{Ph} . \mathrm{D}$. degree in EE from Texas A\&M University, College Station, TX, in 1997. From 1989 to 2004 Dr Qaraqe has held a variety positions in many companies and he has over 12 years of experience in the telecommunication industry. He has worked for Qualcomm, Enad Design Systems, Cadence Design Systems/Tality Corporation, STC, SBC and Ericsson. He has also worked on numerous GSM, CDMA, WCDMA projects and has experience in product development, design, deployments, testing and integration. Dr Qaraqe joined the department of Electrical Engineering of Texas A\&M University at Qatar, in July 2004, where he is now associate professor.

Dr Qaraqe research interests include communication theory and its application to design and performance, analysis of cellular systems and indoor communication systems. Particular interests are in the development of 3G UMTS, cognitive radio systems, broadband wireless communications and diversity techniques.

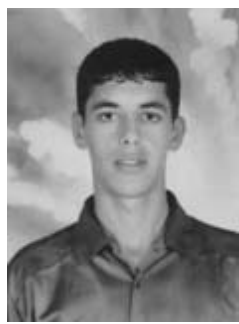

Zied Bouida Received the 'Diplôme d'Ingénieur' degree from École Supérieure des Communications de Tunis (Sup'Com), Tunis, Tunisia, in June 2006. $\mathrm{He}$ received the M.S. degree in Electrical Engineering from Texas A\&M University (TAMU), College Station, TX, in August 2009. He is currently pursuing the $\mathrm{Ph} . \mathrm{D}$. degree in the department of Electrical and Computer Engineering at Texas A\&M University. His research interests include wireless communications, diversity techniques, and cognitive bidirectional relaying.

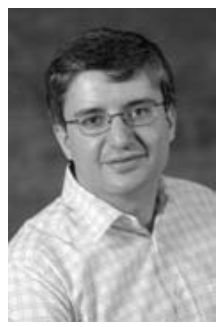

Mohamed-Slim Alouini (S'94, M'98, SM'03, F'09) was born in Tunis, Tunisia. He received the Ph.D. degree in electrical engineering from the California Institute of Technology (Caltech), Pasadena, CA, USA, in 1998. He was with the department of Electrical and Computer Engineering of the University of Minnesota, Minneapolis, MN, USA, then with the Electrical and Computer Engineering Program at the Texas A\&M University at Qatar, Education City, Doha, Qatar. Since June 2009, he has been a Professor of Electrical Engineering in the Division of Physical Sciences and Engineering at KAUST, Saudi Arabia., where his current research interests include the design and performance analysis of wireless communication systems. 